\title{
Ciprofloxacin-resistant Escherichia coli in Central Greece: mechanisms of resistance and molecular identification
}

\author{
Angeliki Mavroidi', Vivi Miriagou ${ }^{2}$, Apostolos Liakopoulos ${ }^{1}$, Eva Tzelepi ${ }^{2}$, Angelos Stefos ${ }^{3}$, George N Dalekos ${ }^{3}$ \\ and Efthymia Petinaki ${ }^{1,4^{*}}$
}

\begin{abstract}
Background: Fluoroquinolone resistant $E$. coli isolates, that are also resistant to other classes of antibiotics, is a significant challenge to antibiotic treatment and infection control policies. In Central Greece a significant increase of ciprofloxacin-resistant Escherichia coli has occurred during 2011, indicating the need for further analysis.

Methods: A total of 106 ciprofloxacin-resistant out of $505 \mathrm{E}$. coli isolates consecutively collected during an eight months period in a tertiary Greek hospital of Central Greece were studied. Antimicrobial susceptibility patterns and mechanisms of resistance to quinolones were assessed, whereas selected isolates were further characterized by multilocus sequence typing and $\beta$-lactamase content.

Results: Sequence analysis of the quinolone-resistance determining region of the gyrA and parC genes has revealed that $63 \%$ of the ciprofloxacin-resistant E. coli harbored a distinct amino acid substitution pattern (GyrA:S83L + D87N; ParC:S80I + E84V), while 34\% and 3\% carried the patterns GyrA:S83L + D87N; ParC:S80I and GyrA:S83L + D87N; ParC:S80I + E84G respectively. The aac (6')-16-cr plasmid-mediated quinolone resistance determinant was also detected; none of the isolates was found to carry the gnrA, anrB and gnrs.

Genotyping of a subset of 35 selected ciprofloxacin-resistant $E$. coli by multilocus sequence typing has revealed the presence of nine sequence types; ST131 and ST410 were the most prevalent and were exclusively correlated with hospital and health care associated infections, while strains belonging to STs 393, 361 and 162 were associated with community acquired infections. The GyrA:S83L + D87N; ParC:S80I + E84V substitution pattern was found exclusively among ST131 ciprofloxacin-resistant E. coli. Extended-spectrum $\beta$-lactamase-positive ST131 ciprofloxacin-resistant isolates produced CTX-M-type enzymes; eight the CTX-M-15 and one the CTX-M-3 variant. CTX-M-1 like and KPC-2 enzymes were detected in five and four ST410 ciprofloxacin-resistant $E$. coli isolates, respectively.
\end{abstract}

Conclusions: Our findings suggest that, ST131 and ST410 predominate in the ciprofloxacin resistant E. coli population.

Keywords: Escherichia coli, Quinolones,MLST, Beta lactamases

\section{Background}

Escherichia coli is among the major pathogens in both community and hospital settings [1]. The prevalence of multidrug-resistant $E$. coli, (i.e., E. coli isolates resistant to more than three classes of antimicrobial agents) has been increased worldwide in the past decades. The emergence

\footnotetext{
* Correspondence: petinaki@med.uth.gr

'Department of Microbiology, University Hospital of Larissa, Larissa, Greece ${ }^{4}$ Department of Microbiology, Medical School, University of Thessaly, Biopolis, Larissa, Greece

Full list of author information is available at the end of the article
}

and worldwide dissemination of fluoroquinolone resistant $E$. coli isolates, that are also resistant to newer $\beta$-lactams due to the production of extended-spectrum $\beta$-lactamases (ESBLs) particularly CTX-M-type enzymes, is a significant challenge to antibiotic treatment and infection control policies [1]. The application of multilocus sequence typing (MLST) to isolates producing CTX-M-15 ESBL led to the recognition of an internationally disseminated clone, ST131, which is a virulent phylogroup B2, uropathogenic E. coli lineage. ST131 E. coli is associated with resistance to fluoroquinolones and aminoglycosides.

\section{Biomed Central}


Quinolones are widely used antimicrobials for the treatment of bacterial infections [2]. Their wide use has triggered increased bacterial resistance worldwide. Mutations in gyrA and parC genes are the most common mechanism involved in high-level quinolone resistance, yet the spread of plasmid- mediated quinolone resistance genes and efflux-pump mutants have also been described. In Greece, according to the recent data of WHONET the rate of ciprofloxacin resistant (CIP-R) E. coli varied from hospital to hospital and ranged from 5.6 to $49.5 \%$. In the University Hospital of Larissa (UHL), that is the main tertiary hospital of Central Greece and serves a region of $1,000,000$ habitants, an increase of ciprofloxacin-resistant E. coli from $16.3 \%$ in 2010 to $21 \%$ in 2011 was recorded. The aim of this study was to assess the epidemiological traits, mechanisms of resistance to fluoroquinolones, phylogenetic relationship and co-existing mechanism of resistance to newer $\beta$-lactams of CIP-R E. coli strains isolated in our institution during 2011.

\section{Methods}

\section{Bacterial isolates,susceptibility testing and clinical data}

From May to December 2011, a total of 505 consecutive E. coli isolates were recovered from clinical samples taken as part of standard care of an equal number of individual patients admitted to the University Hospital of Larissa, Central Greece. Identification and antimicrobial susceptibility testing of bacterial isolates was firstly performed by the Vitek-2 system (bioMérieux, Marcy l'Étoile, France). The interpretive criteria of the European Committee on Antimicrobial Susceptibility Testing were used (http://eucast.org/clinical_breakpoints).

Determination of MICs to ciprofloxacin norfloxacin, imipenem and meropenem was performed by the Etest method (bioMérieux). The double disk synergy test (DDST) was used to determine the ESBL production, as described previously [3]. The modified Hodge test was used for phenotypic detection of carbapenemase production [3]. All E. coli, that were classified as resistant to ciprofloxacin (MICs $>=4 \mathrm{mg} / \mathrm{L}$ ), were further analyzed for the underlying mechanisms of quinolone resistance, molecular typing and $\beta$-lactamase content.

The medical records of patients diagnosed with CIP-R E. coli infection were reviewed regarding their current and previous hospitalizations. Before obtaining the clinical information of the patients, approval was received by the Ethics Committee of the UHL, that is represented by the Infection Control Committee (number of permission 1234). Infections developed $48 \mathrm{~h}$ after hospital admission were characterized as UHL hospital-acquired infections. Infections due to CIP-R E. coli diagnosed within $48 \mathrm{~h}$ of hospital admission were characterized as community acquired infections. Finally, infections in patients who had been hospitalized in the preceding
6 months for more than $48 \mathrm{~h}$ in hospital facilities or nursing homes such as infections in patients transmitted to UHL from other hospitals were considered as health care associated infections.

\section{PCR and sequencing of the Quinolone Resistance- Determining Region (QRDR) of the gyrA and parC genes} All Cip-R E. coli were screened for the presence of mutations in the QRDR of the gyrA and parC genes. After DNA extraction by using the Quick-gDNA TM MiniPrep kit (ZYMO RESEARCH Corp., USA), the QRDRs of both gyrA and parC genes were amplified by polymerase chain reaction (PCR), as described previously [4] and the amplicons were sequenced on both DNA strands using an ABI3730 DNA sequencer (Applied Biosystems, Warrington, United Kingdom). For each isolate, the sequences of the gyrA and $\operatorname{parC}$ gene fragments were concatenated, maintaining the +1 reading frame, aligned and a neighbor-joining tree was constructed from the aligned sequences using the MEGA software [5].

\section{Detection of plasmid-mediated quinolone resistance genes $q n r A, q n r B, q n r S$ and $a a c\left(6^{\prime}\right)-l b-c r$ variant}

Primers and conditions for PCR amplification of the $q n r A$, $q n r B$ and $q n r S$ genes, which encode three targetprotecting proteins and the $a a c\left(6^{\prime}\right)-I b-c r$ variant, which encodes a bifunctional aninoglycoside- fluoroquinolone modified enzyme, were used as described previously [6], and the amplified PCR products obtained were sequenced on both DNA strands as described previously.

\section{Detection of beta-lactamases}

Detection of the bla genes was performed by PCR using a panel of specific primers for $b l a_{\mathrm{TEM}-1}, b l a_{\mathrm{OXA}-1}$, $b l a_{\mathrm{SHV}}, b l a_{\mathrm{CTX}-\mathrm{M}}, b l a_{\mathrm{CMY}}, b l a_{\mathrm{VIM}}$ and $b l a_{\mathrm{KPC}}$, as described previously [7]. PCR products were purified by using the PureLink $^{\mathrm{TM}}$ PCR Purification Kit (Invitrogen, USA) kit and sequenced. Nucleotide and deduced protein sequences were identified by comparing the sequences of the database of G. Jacoby and K. Bush (http://www.lahey.org/ Studies).

\section{Molecular typing of isolates}

The major phylogenetic groups (A, B1, B2, D) were determined by PCR amplification of the three gene fragments of the scheme (chuA, yjaA and TSPE4.C2). Phylogroups were determined as described previously [8]. MLST was performed by PCR amplification and sequencing of seven housekeeping genes: $a d k$ (adenylate kinase), fum $C$ (fumarate hydratase), gyrB (DNA gyrase), icd (isocitrate/ isopropylmalate dehydrogenase), $m d h$ (malate dehydrogenase), purA (adenylosuccinate dehydrogenase), recA (ATP/GTP binding motif) by using primers and conditions as described at the 
MLST Databases at the ERI, University College Cork [9]; http://mlst.ucc.ie/mlstdbs/E.coli. Sequences were obtained on both DNA strands, and alleles and STs were compared with those assigned at the MLST website. Non overlapping groups of related STs were identified using eBURST, with the default setting for the definition of groups [10]; http://eburst.mlst.net.

\section{Results}

One hundred six out 505 (21\%) E. coli isolates were found to be resistant to quinolones. These isolates were obtained from various clinical specimens; mainly from urine (76 out of $106 ; 72 \%$ ) and blood (12 out of 106 ; $11 \%)$, but also from bronchial secretions, cutaneous lessions and sputum. Among them, 30\% (32 isolates) were recovered from community acquired infections, 15\% (16 isolates) from UHL hospital acquired infections and 55\% (58 isolates) from health care associated infections.

Among 106 CIP-R E. coli isolates, 57\% showed an ESBL phenotype, exhibiting resistance to penicillins, expanded- spectrum cephalosporins and aztreonam, while, $4.7 \%$ exhibited also resistance to at least one carbapenem, $72 \%$ were resistant to trimethoprim- sulfamethoxazole, $70 \%$ to tetracycline, $60 \%$ to tobramycin, whereas, only $25 \%$ to gentamicin. Nineteen percent of the CIP-R E. coli isolates exhibited concurrent resistance to four other classes of antimicrobial agents, $58.5 \%$ to three classes, $13.2 \%$ to two classes and $3.8 \%$ to one class; the remaining $5.5 \%$ showed resistance only to quinolones.

Sequencing of the QRDR regions of the gyrA and parC genes of CIP- $\mathrm{R}$ isolates has revealed three different amino acid substitution patterns: GyrA:S83L + D87N; ParC:S80I + E84V ( $\mathrm{n}=67,63 \%)$, GyrA:S83L + D87N; ParC:S80I $(n=36,34 \%)$ and GyrA:S83L + D87N; ParC: $\mathrm{S} 80 \mathrm{I}+\mathrm{E} 84 \mathrm{G}(\mathrm{n}=3,3 \%)$. All but one of the 67 CIP-R $E$. coli isolates, that possessed the GyrA:S83L + D87N; ParC:S80I + E84V pattern, showed identical nucleotide sequences in the gyr $A$ and parC, with the exception showing only a synonymous nucleotide substitution in the parC gene. In addition, the three isolates with the GyrA:S83L + D87N; ParC:S80I + E84G pattern had identical nucleotide sequences. On the contrary, the nucleotide sequences of gyrA/ parC gene fragments of the 36 CIP-R isolates with the GyrA:S83L + D87N; ParC:S80I substitution pattern were found to be more polymorphic.

Out of the 106 CIP-R coli isolates, 35 were selected for further investigation including 21 with the pattern GyrA: S83L + D87N; ParC:S80I + E84V, 13 with the pattern GyrA:S83L + D87N; ParC:S80I, and one with the pattern GyrA:S83L + D87N; ParC:S80I + E84G. The selection was designed so as to include proportionally all the variations in the amino and nucleotide acid substitution patterns observed in the GyrA and ParC, the antimicrobial resistance patterns, the origin and the distribution of the isolates over the study period (Table 1).

The 35 representative CIP-R E.coli isolates were distributed into phylogroups B2 (30 isolates), D (two isolates), A (two isolates) and B1 (one isolate) [Table 1]. Genotyping by MLST has revealed the presence of nine STs. ST131 (21 out 35 isolates), and ST410 (six out 35 isolates) were the most prevalent; ST44, ST90, ST162, ST361, ST1140, ST2509 included one isolate, while, ST393 included two isolates. According to clinical data, all ST131 isolates were associated with UHL hospital acquired infections and health care associated infections, ST410 CIP-R E. coli were exclusively associated with health care associated infections, while STs 393, 361 and 162 from community acquired infections. Strains that belonged to ST90, 44, 1140 and 2509 were isolated from patients with UHL hospital acquired infections.

Although, only three amino acid substitution patterns in the GyrA/ParC were identified among the CIP-R E. coli, we have sought to investigate any possible correlations of the nucleotide polymorphisms and the STs of the isolates. For this purpose, a neighbor-joining tree was constructed from the concatenated sequences of the gyrA/ parC gene fragments (Figure 1). The pattern GyrA:S83L + D87N;ParC:S80I + E84V was associated with ST131 strains possessing identical nucleotide sequences, while, the GyrA:S83L + D87N; ParC:S80I was found to various STs, including ST410. Except from one isolate (ID 362), the rest isolates with this substitution pattern differed in 12 polymorphic sites of the $643 \mathrm{bp}$ nucleotide sequence of the gyrA/parC fragments. The third pattern, GyrA:S83L + D87N; ParC:S80I + E84G, was associated with ST393 (ID 296). The isolate ID 362, that belonged also to ST393, showed similarity in the gyrA/ parC nucleotide sequences with the isolate ID 296 and they differed at only a single nucleotide site.

The presence of plasmid- mediated quinolone resistance genes was also investigated in the 35 CIP-R E.coli isolates (Table 1, Figure 1). The aac (6')- $1 b-c r$ variant was detected in 23 out of 35 CIP-R E.coli; of these 14 were ST131 and five ST410. The remaining four isolates belonged to ST44, ST90 and ST162 and ST393. None isolate was found to carry any of the $q n r A, q n r B$ and qnrS gene.

The $\beta$-lactamase content of the 35 Cip-R E. coli was also determined (Table 1). ESBL- positive CIP-R isolates were found to produce enzymes of the CTX-M-1 family $(\mathrm{n}=15)$; CTX-M-15 $(\mathrm{n}=12)$ and CTX-M-3 $(\mathrm{n}=3)$. Among them, nine belonged to ST131, five to ST410 and one to ST44 (Table 1). One out of the twelve ESBL-negative ST131 was a VIM-1 producer. Three ST410 CTX-M-producing strains co-produced the KPC2 carbapenemase. 
Table 1 Microbiological characteristics of the 35 representative CIP-R E. coli; phylogroups, MLST STs (clonal complexes; Cplx), presence/absence of the aac (6')-1b-cr variant and beta-lactamase content

\begin{tabular}{|c|c|c|c|c|c|}
\hline $\begin{array}{l}\text { Strain } \\
\text { ID }\end{array}$ & $\begin{array}{l}\text { Isolation } \\
\text { date }\end{array}$ & Antimicrobial resistant pattern & ESBL & $\begin{array}{l}\text { Phylogroup/ MLST } \\
\text { ST (CC) }\end{array}$ & $\begin{array}{l}\text { aac beta-lactamase content } \\
\left(6^{\prime}\right)-l b-c r\end{array}$ \\
\hline
\end{tabular}

GyrA:S83L + D87N/ ParC: S80I + E84V ( $n=21)$

\begin{tabular}{lllllll}
\hline 347 & $13 / 6 / 2011$ & AM,AMC,CAZ,CTX,ATM,GM,NN,TE,SXT,CIP, NOR & positive & B2/ ST131 & $(-)$ & CTX-M-15 \\
\hline 161 & $27 / 5 / 2011$ & AM,AMC,CAZ,CTX,ATM, NN,TE,SXT,CIP NOR & positive & B2/ ST131 & $(+)$ & CTX-M-15+ OXA-1 \\
\hline 391 & $30 / 5 / 2011$ & AM,AMC,CAZ,CTX,ATM,GM,NN,TE,SXT,CIP,NOR & positive & B2/ ST131 & $(+)$ & CTX-M-15+ OXA-1 \\
\hline 392 & $9 / 6 / 2011$ & AM,AMC,CAZ,CTX,ATM,GM,NN,TE,SXT,CIP,NOR & positive & B2/ ST131 & $(+)$ & CTX-M-15+ OXA-1 \\
\hline 393 & $18 / 7 / 2011$ & AM,AMC,CAZ,CTX,ATM,GM,NN,TE,SXT,CIP,NOR & positive & B2/ ST131 & $(+)$ & CTX-M-15+ OXA-1 \\
\hline 399 & $2 / 8 / 2011$ & AM,AMC,CAZ,CTX,ATM,GM,NN,TE,SXT,CIP,NOR & positive & B2/ ST131 & $(+)$ & CTX-M-15+ OXA-1 \\
\hline 160 & $3 / 5 / 2011$ & AM,AMC,CAZ,CTX,ATM,SXT,CIP,NOR & positive & B2/ ST131 & $(+)$ & CTXM-15+ TEM-1 \\
\hline 505 & $4 / 9 / 2011$ & AM,AMC,CAZ,CTX,ATM,AN,GM,NN,SXT,CIP,NOR & positive & B2/ ST131 & $(+)$ & CTX-M-15+ TEM-1 + OXA-1 \\
\hline 397 & $4 / 8 / 2011$ & AM,AMC,CAZ,CTX,ATM,TE, SXT, CIP, NOR & positive & B2/ ST131 & $(-)$ & CTX-M-3 \\
\hline 270 & $1 / 5 / 2011$ & AM,AMC,NN,SXT,CIP,NOR & negative & B2/ ST131 & $(+)$ & OXA-1 \\
\hline 301 & $5 / 5 / 2011$ & AM, AMC,NN,TE,CIP,NOR & negative & B2/ ST131 & $(+)$ & OXA-1 \\
\hline 307 & $6 / 6 / 2011$ & AM,AMC, GM, NN, TE,CIP, NOR & negative & B2/ ST131 & $(+)$ & OXA-1 \\
\hline 252 & $1 / 5 / 2011$ & AM, SXT,CIP,NOR & negative & B2/ ST131 & $(-)$ & TEM-1 \\
\hline 206 & $16 / 5 / 2011$ & AM,AMC,GM,TE,SXT,CIP,NOR & negative & B2/ ST131 & $(+)$ & TEM-1 + OXA-1 \\
\hline 320 & $25 / 5 / 2011$ & AM,AMC,CIP,NOR & negative & B2/ ST131 & $(+)$ & TEM-1 + OXA-1 \\
\hline 346 & $3 / 6 / 2011$ & AM,AMC,AN,GM,NN,SXT,TE,CIP,NOR & negative & B2/ ST131 & $(+)$ & TEM-1 + OXA-1 \\
\hline 803 & $4 / 12 / 2011$ & AM,AMC,CAZ,CTX, IMP,MEM,CIP, NOR & negative & B2/ ST131 & $(+)$ & VIM-1-like + TEM-1+ OXA-1 \\
\hline 220 & $5 / 5 / 2011$ & TE, SXT,CIP,NOR & negative & B2/ ST131 & $(-)$ & none \\
\hline 321 & $18 / 7 / 2011$ & CIP,NOR & negative & B2/ ST131 & $(-)$ & none \\
\hline 354 & $1 / 7 / 2011$ & TE,CIP,NOR & negative & B2/ ST131 & $(-)$ & none \\
\hline 355 & $15 / 8 / 2011$ & TE,SXT,CIP,NOR & negative & B2/ ST131 & $(-)$ & none \\
\hline
\end{tabular}

GyrA:S83L + D87N/ ParC: S80I + E84G $(n=1)$

\begin{tabular}{|c|c|c|c|c|c|c|}
\hline 296 & $6 / 8 / 2011$ & $\mathrm{AM}, \mathrm{TE}, \mathrm{CIP}, \mathrm{NOR}$ & negative & $\begin{array}{l}\text { D/ ST393 (ST31 } \\
\text { Cplx) }\end{array}$ & $(+)$ & TEM-1 \\
\hline $\begin{array}{l}\text { Strain } \\
\text { ID }\end{array}$ & $\begin{array}{l}\text { Isolation } \\
\text { date }\end{array}$ & Antimicrobial susceptibility pattern & ESBL & $\begin{array}{l}\text { Phylogroup/MLST } \\
\text { ST (CC) }\end{array}$ & $\begin{array}{l}a a c \\
\left(6^{\prime}\right)-l b-c r\end{array}$ & beta-lactamase content \\
\hline \multicolumn{7}{|c|}{ GyrA:S83L + D87N/ ParC: S80I $(n=13)$} \\
\hline \multirow[t]{2}{*}{148} & \multirow[t]{2}{*}{ 9/6/2011 } & \multirow{2}{*}{$\begin{array}{l}\text { AM,AMC,CAZ,CTX,ATM,IMP,MEM,NN,SXT,CIP, } \\
\text { NOR }\end{array}$} & \multirow[t]{2}{*}{ positive } & $\mathrm{B} 2 / \mathrm{ST} 410$ & \multirow[t]{2}{*}{$(+)$} & \multirow{2}{*}{$\begin{array}{l}\text { KPC-2 + CTXM-15 + TEM-1 + } \\
\text { OXA-1 }\end{array}$} \\
\hline & & & & (ST23 Cplx) & & \\
\hline \multirow[t]{2}{*}{182} & \multirow[t]{2}{*}{$17 / 5 / 2011$} & \multirow{2}{*}{$\begin{array}{l}\text { AM,AMC,CAZ,CTX,ATM,IMP,MEM,AN,NN,TE,CIP, } \\
\text { NOR }\end{array}$} & \multirow[t]{2}{*}{ positive } & B2/ ST410 & \multirow[t]{2}{*}{$(+)$} & \multirow{2}{*}{$\begin{array}{l}\text { KPC-2 + CTXM-3 + TEM-1+ OXA- } \\
1\end{array}$} \\
\hline & & & & (ST23 Cplx) & & \\
\hline \multirow[t]{2}{*}{252} & \multirow[t]{2}{*}{ 10/6/2011 } & \multirow[t]{2}{*}{ AM,AMC,CAZ,CTX,ATM,IMP,MEM,NN,TE,CIP,NOR } & \multirow[t]{2}{*}{ positive } & B2/ ST410 & \multirow[t]{2}{*}{$(+)$} & \multirow{2}{*}{$\begin{array}{l}\text { KPC-2 + CTXM-3 + TEM-1 + OXA } \\
1\end{array}$} \\
\hline & & & & (ST23 Cplx) & & \\
\hline \multirow[t]{2}{*}{648} & \multirow[t]{2}{*}{$31 / 12 / 2011$} & \multirow[t]{2}{*}{ AM,AMC,CAZ,CTX,IMP,MEM,NN,TE,CIP,NOR } & \multirow[t]{2}{*}{ negative } & B2/ ST410 & \multirow[t]{2}{*}{$(-)$} & \multirow[t]{2}{*}{$\mathrm{KPC}-2+\mathrm{TEM}-1$} \\
\hline & & & & (ST23 Cplx) & & \\
\hline \multirow[t]{2}{*}{132} & \multirow[t]{2}{*}{ 24/5/2011 } & \multirow[t]{2}{*}{ AM,AMC,CAZ,CTX,ATM,AN,NN,TE,SXT,CIP,NOR } & \multirow[t]{2}{*}{ positive } & B2/ ST410 & \multirow[t]{2}{*}{$(+)$} & \multirow[t]{2}{*}{ CTXM-15 + TEM-1 + OXA-1 } \\
\hline & & & & (ST23 Cplx) & & \\
\hline \multirow[t]{2}{*}{281} & \multirow[t]{2}{*}{$15 / 8 / 2011$} & \multirow{2}{*}{$\begin{array}{l}\text { AM,AMC,CAZ,CTX,ATM,AN,GM,NN,TE,SXT,CIP, } \\
\text { NOR }\end{array}$} & \multirow[t]{2}{*}{ positive } & $\mathrm{B} 2 / \mathrm{ST} 410$ & $(+)$ & CTXM-15+ OXA-1 \\
\hline & & & & (ST23 Cplx) & & \\
\hline 384 & $30 / 11 / 2011$ & AM,AMC,CAZ,CTX,ATM,AN,TE,SXT,CIP,NOR & positive & B2/ ST44 & $(+)$ & CTXM-15 \\
\hline & & & & (ST10 Cplx) & & \\
\hline 383 & $22 / 9 / 2011$ & $\mathrm{AM}, \mathrm{TE}, \mathrm{SXT}, \mathrm{CIP}, \mathrm{NOR}$ & negative & A/ ST90 & $(+)$ & TEM-1 \\
\hline & & & & (ST23 Cplx) & & \\
\hline 259 & $14 / 7 / 2011$ & $\mathrm{AM}, \mathrm{TE}, \mathrm{SXT}, \mathrm{CIP}, \mathrm{NOR}$ & negative & B1/ ST162 & $(+)$ & TEM-1 \\
\hline & & & & (ST469 Cplx) & & \\
\hline
\end{tabular}


Table 1 Microbiological characteristics of the 35 representative CIP-R E. coli; phylogroups, MLST STs (clonal complexes; Cplx), presence/absence of the aac $\left(6^{\prime}\right)-1 b$-cr variant and beta-lactamase content (Continued)

\begin{tabular}{lllllll}
\hline 328 & $10 / 9 / 2011$ & AM,SXT,CIP,NOR & negative & B2/ ST361 & $(-)$ & TEM-1 \\
\hline 362 & $21 / 10 / 2011$ & AM,TE,SXT,CIP,NOR & negative & D/ ST393 & $(-)$ & TEM-1 \\
& & & (ST31 Cplx) & & \\
\hline 129 & 25/5/2011 & AM,TE,SXT,CIP,NOR & negative & B2/ ST1140 & $(-)$ & TEM-1 \\
\hline 327 & $9 / 6 / 2011$ & CIP, NOR & negative & A/ ST2509 & $(-)$ & none \\
\hline
\end{tabular}

\section{Discussion}

During an eight months period of 2011, CIP-R E. coli accounted for $21 \%$ of the total $E$. coli isolates recovered from various clinical specimens from outpatients and inpatients of the UHL. The majority of the CIP-R E. coli isolates were multidrug-resistant, posing a challenge for therapeutic options. Only $5.5 \%$ were resistant to fluoroquinolones, but susceptible to various antimicrobial classes. The latter strains were recovered from both community and hospital acquired infections.

The majority of CIP-R E. coli belonged to ST131 and ST410, which were recovered from hospital and health care associated infections, whereas other studies have shown that such strains were disseminated in the community [11-16]. We have also shown previously that ST410 was linked with an outbreak of KPC-producing E. coli in a long-term care facility unit of Thessalia [17]; this clone, apart from carbapenemase producers, includes also isolates with CTX-M-15, as it was firstly reported in Spain [16]. All ST131 and ST410 CIP-R E. coli belonged to the virulent phylogroup $\mathrm{B} 2$, which is associated epidemiologically and experimentally with extraintestinal virulence $[12,18]$. The remaining isolates, that were isolated in a sporadic fashion in our study, were distributed equally to various STs, which have been previously reported among Cip-R E. coli isolates, and originated from community and hospital acquired infections [15]. 
In Greece, there are few studies on the mechanisms of resistance to fluoroquinolones [19-21], but to our knowledge this is the first report on the association of gyrA and $\operatorname{parC}$ mutations and the phylogenetic lineages of ciprofloxacin-resistant E. coli in Greece. According to our results, ST131 and ST410 carried specific patterns of GyrA/ParC amino substitutions. In more details, ST131 Cip-R E. coli in our hospital possessed the same amino acid substitution pattern GyrA:S83L + D87N; ParC:S80I + E84V, which has been previously identified among ST131 CIP-R E. coli isolated from humans and companion animals in the United States, United Kingdom, Australia and Korea [13-15]. On the other hand, the six ST 410 E. coli of our study carried the GyrA: S83L + D87N; ParC:S80I pattern, which has been identified in previous studies [22,23]. Nevertheless, the sequence types of the latter strains have not been determined.

The presence of plasmid-mediated resistance genes ( $q n r A, q n r B$ and $q n r S$ ) has also been reported in previous studies in Greece [19-21], but these genes were not detected in our collection of CIP-R E. coli isolates. On the other hand, the $a a c$ (6')-1b-cr variant was detected mainly in strains belonging to both ST131 and ST410, a finding that was also previously described $[13,16]$.

Since isolates possessing the GyrA:S83L + D87N; ParC:S80I + E84V comprised a high percentage (67\%) among CIP-R E. coli, and the representative isolates of this group have been assigned to ST131, we assume that the increase of fluoroquinolone-resistance during 2011 can be attributed to the dissemination of ST131 strains. The source and the time of importation of these isolates are unknown. As mentioned previously, since ST131 was first described in 2008 [24], it has disseminated worldwide [11-15,23-26]. Several factors such as host-tohost or foodborne transmission or environmental contamination have been suggested to contribute in their dissemination; reservoirs of ST131 have been identified in food and water sources, in nursing home residents, companion animals and food sources [27-29].

Central Greece is a rural area, where fluoroquinolones are widely used in veterinary and poultry, but no data exist about the incidence of resistant $E$. coli of animal source. It would be interesting to investigate the mechanisms of resistance and genetic relatedness of such strains in future studies. These studies would elucidate the relationships between STs of isolates recovered from human and animals and the roots of transmission.

\section{Conclusions}

In the present study we have characterized the mechanisms of resistance and explored the genetic relatedness of CIP-R E. coli recovered from community, hospital and health care associated infections in a tertiary care hospital in Central Greece. Our findings suggest that ST131 and ST410 predominate in the CIP-R E. coli population in our institution. The increase of resistance to fluoroquinolones observed during 2011 is attributed mainly to the wide dissemination of ST131 CIP-R. E. coli.

\section{Abbreviations}

CIP-R: ciprofloxacin resistant; MLST: Multilocus sequence typing; ST: Sequence type; CC: Clonal complex; ESBL: Extended-spectrum $\beta$-lactamases;

KPC: Klebsiella pneumoniae carbapenemase; QRDR: Quinolone ResistanceDetermining Region; UHL: University Hospital of Larissa; DDST: Double disk synergy test; AM: Amoxicillin; AMC: Amoxicillin + clavulanic acid; CAZ: Ceftazidime; CTX: Cefotaxime; ATM: Aztreonam; GM: Gentamicin; NN: Tobramycin; AN: Amikacin; TE: Tetracycline; SXT: Trimethoprimsulfomethoxazole; CIP: Ciprofloxacin; NOR: Norfloxacin; IMP: Imipenem; MEM: Meropenem

\section{Competing interests}

The authors declare that they have no competing interests.

\section{Authors' contributions}

EP, AM, and VM conceived and designed the study. AM wrote the first draft of the paper and other co-authors contributed to the final draft. EP and GND were responsible for conducting the study and managing the data. AS conducted the interpretation of data. Others participated in data analysis and data interpretation. All authors read and approved the final manuscript.

\section{Author details}

'Department of Microbiology, University Hospital of Larissa, Larissa, Greece. ${ }^{2}$ Laboratory of Bacteriology, Hellenic Pasteur Institute, Athens, Greece. ${ }^{3}$ Department of Medicine, Medical School, University of Thessaly, Larissa, Greece. ${ }^{4}$ Department of Microbiology, Medical School, University of Thessaly, Biopolis, Larissa, Greece.

Received: 3 September 2012 Accepted: 18 December 2012 Published: 23 December 2012

\section{References}

1. Woodford N, Turton JF, Livermore DM: Multiresistant Gram-negative bacteria: the role of high-risk clones in the dissemination of antibiotic resistance. FEMS Microbiol Rev 2011, 35:736-755.

2. Jacoby GA: Mechanisms of resistance to quinolones. Clin Infect Dis 2005, 41(Suppl 2):S120-S126.

3. Thomson KS: Extended-Spectrum- $\beta$-Lactamase, AmpC, and Carbapenemase. J Clin Microbiol 2010, 48:1019-1025.

4. Jurado S, Orden JA, Horcajo P, De La Fuente R, Ruiz-Santa-Quiteria JA Martínez-Pulgarín S, Domínguez-Bernal G: Characterization of fluoroquinolone resistance in Escherichia coli strains from ruminants. J Vet Diagn Invest 2008, 20:342-345.

5. Tamura K, Dudley J, Nei M, Kumar S: MEGA4: Molecular Evolutionary Genetics Analysis (MEGA) software version 4.0. Mol Biol Evol 2007, 24:1596-1599

6. Robicsek A, Strahilevitz J, Sahm DF, Jacoby GA, Hooper DC: anr prevalence in ceftazidime-resistant Enterobacteriaceae isolates from the United States. Antimicrob Agents Chemother 2006, 50:2872-2874.

7. Papagiannitsis CC, Tryfinopoulou K, Giakkoupi P, Pappa O, Polemis M, Tzelepi E, Tzouvelekis LS, Vatopoulos AC: Diversity of acquired $\beta$ lactamases amongst Klebsiella pneumoniae in Greek hospitals. Int J Antimicrob Agents 2012, 39:178-180.

8. Clermont O, Bonacorsi S, Bingen E: Rapid and simple determination of the Escherichia coli phylogenetic group. Appl Environ Microbiol 2000, 66:4555-4558

9. Wirth T, Falush D, Lan R, Colles F, Mensa P, Wieler LH, Karch H, Reeves PR, Maiden MCJ, Ochman H, Achtman M: Sex and virulence in Escherichia coli: an evolutionary perspective. Mol Microbiol 2006, 60:1136-1151.

10. Feil EJ, Li BC, Aanensen DM, Hanage WP, Spratt BG: eBURST: inferring patterns of evolutionary descent among clusters of related bacterial genotypes from multilocus sequence typing data. J Bacterio/ 2004, 186:1518-1530. 
11. Rogers BA, Sidjabat HE, Paterson DL: Escherichia coli O25b-ST131: a pandemic, multiresistant, community-associated strain. J Antimicrob Chemother 2011, 66:1-14.

12. Johnson JR, Johnson B, Clabots C, Kuskowski MA, Castanheira M: Escherichia coli sequence type ST131 as the major cause of serious multidrugresistant E. coli infections in the United States. Clin Infect Dis 2010, 51:234-286

13. Platell JL, Cobbold RN, Johnson JR, Heisig A, Heisig P, Clabots C, Kuskowsk MA, Trott DJ: Commonality among fluoroquinolone-resistant sequence type ST131 extraintestinal Escherichia coli isolates from humans and companion animals in Australia. Antimicrob Agents Chemother 2011, 55:3782-3787

14. Clark G, Paszkiewicz K, Hale J, Weston V, Constantinidou C, Penn C, Achtman M, McNally A: Genomic analysis uncovers a phenotypically diverse but genetically homogeneous Escherichia coli ST131 clone circulating in unrelated urinary tract infections. J. Antimicrob. Agents 2012, 67:868-877.

15. Lee MY, Choi HJ, Choi JY, Song M, Song Y, Kim S-W, Chang H-H, Jung S-I, Kim Y-S, Ki HK, Son JS, Kwon KT, Heo ST, Yeom J-S, Shin SY, Chung DR, Peck KR, Song J-H, Ko KS: Dissemination of ST131 and ST393 communityonset, ciprofloxacin-resistant Escherichia coli clones causing urinary tract infections in Korea. J Infect 2010, 60:146-153.

16. López-Cerero L, Egea P, Serrano L, Navarro D, Mora A, Blanco J, Doi Y, Paterson DL, Rodríguez-Baño J, Pascual A: Characterisation of clinical and food animal Escherichia coli isolates producing CTX-M-15 extendedspectrum $\beta$-lactamase belonging to ST410 phylogroup A. Int J Antimicrob Agents 2011, 37:365-367.

17. Mavroidi A, Miriagou V, Malli E, Stefos A, Dalekos GN: Emergence of Escherichia coli sequence type 410 (ST410) with KPC-2 $\beta$-lactamase. Int J Antimicrob Agents 2012, 39:247-250.

18. Le Gall T, Clermont O, Gouriou S, Picard B, Nassif X, Denamur E, Tenaillon O: Extraintestinal virulence is a coincidental by-product of commenalism in B2 phylogenetic group Escherichia coli strains. Mol Biol Evol 2007, 24:2373-2384

19. Chaniotaki S, Giakouppi P, Tzouvelekis LS, Panagiotakos D, Kozanitou M, Petrikkos G, Avlami A, Vatopoulos AC, WHONET Study Group: Quinolone resistance among Escherichia coli strains from community-acquired urinary tract infections in Greece. Clin Microbiol Infect 2004, 10:75-78.

20. Vasilaki O, Ntokou E, Ikonomidis A, Sofianou D, Frantzidou F, Alexiou-Daniel S, Maniatis AN, Pournaras S: Emergence of the plasmid-mediated quinolone resistance gene qnrS1 in Escherichia coli isolates in Greece. Antimicrob Agents Chemother 2008, 52:2996-2997.

21. Galani I, Souli M, Mitchell N, Chryssouli Z, Giamarellou H: Presence of plasmid-mediated quinolone resistance in Klebsiella pneumoniae and Escherichia coli isolates possessing blaVIM-1 in Greece. Int J Antimicrob Agents 2010, 36:252-254

22. Morgan-Linnell SK, Lauren Becnel B, David S, Zechiedrich L: Mechanisms accounting for fluoroquinolone resistance in Escherichia coli clinical isolates. Antimicrob Agents Chemother 2009, 53(1):235-241.

23. Cagnacci S, Gualco L, Debbia E, Schito GC, Marchese A: European emergence of ciprofloxacin-resistant Escherichia coli clonal groups 025 : H4-ST 131 and 015:K52:H1 causing community-acquired uncomplicated cystitis. J Clin Microbiol 2008, 46:2605-2612.

24. Coque TM, Novais A, Carattoli A, Poirel L, Pitout J, Peixe L, Baquero F, Cantón R, Nordmann P: Dissemination of clonally related Escherichia coli strains expressing extended-spectrum beta-lactamase CTX-M-15. Emerg Infect Dis 2008, 14:195-200

25. Blanco J, Mora A, Mamani R, López C, Blanco M, Dahbi G, Herrera A, Blanco JE, Alonso MP, García-Garrote F, Chaves F, Orellana MÁ, Martínez-Martínez L, Calvo J, Prats G, Larrosa MN, González-López JJ, López-Cerero L, RodríguezBaño J, Pascual A: National survey of Escherichia coli causing extraintestinal infections reveals the spread of drug-resistant clonal groups O25b:H4-B2-ST131, O15:H1-D-ST393 and CGA-D-ST69 with high virulence gene content in Spain. J Antimicrob Chemother 2011 , 66:2011-2021.

26. Oteo J, Diestra K, Juan C, Bautista V, Novais A, Pérez-Vázquez M, Moyá B, Miró E, Coque TM, Oliver A, Cantón R, Navarro F, Campos J: Extendedspectrum beta-lactamase-producing Escherichia coli in Spain belong to a large variety of multilocus sequence typing types, including ST10 complex/A, ST23 complex/A and ST131/B2. Int J Antimicrob Agents 2009, 34:173-176.
27. Sebastian Guenther S, Ewers C, Wieler LH: Extended-spectrum beta lactamases producing E.coli in wildlife, yet another form of environmental pollution? Front Microbiol 2011, 2:246.

28. Giufrè M, Graziani C, Accogli M, Cerquetti M: Food reservoir for Escherichia coli causing urinary tract infections. Emerg Infect Dis 2010, 16:1048-1049.

29. Vincent C, Boerlin P, Daignault D, Dozois CM, Dutil L, Galanakis C, ReidSmith RJ, Tellier P-P, Tellis PA, Ziebell K, Manges AR: Food reservoir for Escherichia coli causing urinary tract infections. Emerg Infect Dis 2010, 16:88-95.

doi:10.1186/1471-2334-12-371

Cite this article as: Mavroidi et al:: Ciprofloxacin-resistant Escherichia coli in Central Greece: mechanisms of resistance and molecular identification. BMC Infectious Diseases 2012 12:371.

\section{Submit your next manuscript to BioMed Central and take full advantage of:}

- Convenient online submission

- Thorough peer review

- No space constraints or color figure charges

- Immediate publication on acceptance

- Inclusion in PubMed, CAS, Scopus and Google Scholar

- Research which is freely available for redistribution

Submit your manuscript at www.biomedcentral.com/submit
C) Biomed Central 